\title{
Análise de linfonodos no câncer colorretal: 0 impacto do uso de uma solução clareadora de gorduras
}

Primeira submissão em 20/06/07 Última submissão em 20/12/08 Aceito para publicação em 06/12/08 Publicado em 20/12/08

\section{Lymph node analysis in colorectal cancer: the impact of the use of fat clearance solution}

Rair Geraldo Richard Xavier'; Túlio Pereira Júnior²; Ana Margarida M. F. Nogueira (in memoriam)³

\section{unitermos}

Carcinoma colorretal

Metástases linfonodais

Estadiamento

Solução clareadora de gordura

\section{resumo}

Introdução: O acometimento de linfonodos (LN) é o principal fator prognóstico em carcinomas colorretais (CCR) e recomendam-se técnicas especiais para melhorar a análise dos LN. O objetivo deste trabalho foi avaliar o impacto do uso de uma solução clareadora de gorduras na dissecção de LN em CCR. Material e método: Analisaram-se prospectivamente 74 adenocarcinomas (35 homens, média de 62,9 anos), a maioria pT3. Após o exame de rotina e dissecção de LN pelo método convencional (MC), a gordura foi imersa por 36 h (três trocas) em uma solução (SC) composta por álcool (65\%), éter (20\%), ácido acético (5\%) e formol (10\%) e realizou-se nova dissecção. Mediu-se o diâmetro dos LN e determinou-se o número de $\mathrm{LN}$ positivos $(\mathrm{LN}+)$ e negativos $<4 \mathrm{~mm}$. Alguns $\mathrm{LN}+$ obtidos após a $\mathrm{SC}$ foram submetidos à imunomarcação por AE1/AE3. Resultados: Dissecaram-se 3.167 LN (42,8/caso): 1.814 (24,5/caso) pelo MC e 1.355 (18,3/caso) após o uso da SC; detectaram-se $196 \mathrm{LN}+170$ (86,7\%) pelo MC e $26(13,2 \%)$ após a SC; $1.068 \mathrm{LN}$ (59\%) dissecados convencionalmente eram $<4 \mathrm{~mm}$ (variando de 0,4-23 mm) e 1.261 LN (93\%) dissecados após a SC eram < $4 \mathrm{~mm}$ (variando de 0,2-11 mm); 39/170 (22,9\%) LN+ dissecados convencionalmente tinham menos de $4 \mathrm{~mm}$ e após a SC 19/26 (73\%) dos $\mathrm{LN}+$ eram $<4 \mathrm{~mm}(p=0)$. 0 uso da SC aumentou o número de LN em $74,7 \%(p=0)$, a detecção de $\mathrm{LN}$ pequenos em $118 \%(p=0)$ e a detecção de metástases em $15,3 \%(p=0,003)$. Houve modificação do $\mathrm{pN}$ em cinco casos $(p=0,03)$ nos quais se dissecaram inicialmente $12-20 \mathrm{LN} ; 90 \%$ dos casos com $\mathrm{LN}+$ foram detectados somente após a análise de $40 \mathrm{LN}$. Conclusão: É necessário analisar grande número de LN, principalmente os pequenos, para estadiar adequadamente o carcinoma colorretal. Uma solução de clareamento de gorduras simples de usar e pouco dispendiosa pode ser de grande ajuda nesse propósito.

\section{abstract}

Background: Lymph node ( $L N)$ involvement is the most important prognostic factor in colorectal carcinomas and special methods are recommended to improve $L N$ analysis. The objective of this study was to evaluate the impact of the use of a fat bleaching solution in LN dissection in colorectal carcinomas. Method: 74 adenocarcinomas (35 men - average age: 62.9) were prospectively analysed, most of them were pT3. After routine exam and LN dissection through conventional method, the fat was soaked in a solution containing alcohol (65\%), ether (20\%), acetic acid (5\%) and formalin (10\%) for 36 hours (three changes). A new dissection was performed subsequently. $L N$ diameters were measured and the number of positive $(L N+)$ and negative $L N$ with $<4 \mathrm{~mm}$ was determined. Some LN+ obtained after the bleaching procedure were immunolabelled by AE1/AE3. Results: 3,167 LN(42.8/case) were dissected: 1,814 (24.5/case) by CM and 1,355 (18.3/case) after the bleaching procedure. $196 \mathrm{LN}+$ were detected: 170 (86.7\%) detected by CM and 26 (13.2\%) after the bleaching procedure. 1,068 (59\%) of the LN conventionally dissected were $<4 \mathrm{~mm}$ (range 0.4-23 mm) and 1,261 (93\%) of the LN dissected after the bleaching procedure were $<4 \mathrm{~mm}$ (range 0.2-11 $\mathrm{mm}$ ). 39/170 (22.9\%) LN+ conventionally dissected were $<4 \mathrm{~mm}$ and $19 / 26$ (73\%) of the $L N+$ dissected after the bleaching procedure measured $<4 \mathrm{~mm}(\mathrm{p}=0)$. The use of bleaching solution increased the number of $L N$ by $74.7 \%(p=0.000)$, the detection of small $L N$ by $118 \%(p=0)$ and the detection of metastasis by $15.3 \%(p=0.003)$. There was modification of $p N$ in 5 cases $(p=0.03)$, from which 12-20 LN had been initially examined. $90 \%$ of all $L N+$ cases were detected only after the analysis of $40 \mathrm{LN}$. Conclusion: A large number of $L N$, mainly the small ones, must be analysed to stage colorectal cancer adequately. The use of a fat bleaching solution is inexpensive and may be a highly suitable procedure.

1. Médico patologista; mestrando em Patologia Médica pela Universidade Federal de Minas Gerais (UFMG); bolsista do Conselho Nacional de Desenvolvimento Científico e Tecnológico (CNPq). 2. Médico generalista.

3. Doutora; professora adjunta do Departamento de Anatomia Patológica e Medicina Legal da Faculdade de Medicina da UFMG; bolsista de Produtividade em Pesquisa do CNPq

Trabalho realizado no Departamento de Anatomia Patológica e Medicina Legal, Laboratório de Patologia Castrintestinal, Faculdade de Medicina da UFMG, com base na dissertação de mestrado

"Análise de linfonodos no câncer colorretal: o impacto do uso de uma solução clareadora de gorduras" apresentada ao Curso de Pós-Graduação em Patologia, UFMG, 2007. Apoio Financeiro: CNPq. 


\section{Introdução}

O carcinoma colorretal (CCR) é importante causa de morbidade e mortalidade na população mundial, sendo o terceiro câncer mais comum em mulheres e o quarto em homens ${ }^{(35)}$. Segundo estimativas do Instituto Nacional do Câncer do Brasil para o ano de 2008, o CCR seria o terceiro tumor maligno mais freqüente em homens e o segundo em mulheres na região sudeste do $\mathrm{Brasil}^{(20)}$.

As características anatomopatológicas do tumor são os mais poderosos fatores preditivos para avaliar a evolução pós-cirúrgica no $\mathrm{CCR}^{(6,9)}$. A presença de metástases em linfonodos (LN) regionais é, isoladamente, o fator prognóstico mais importante do CCR e o estadiamento patológico dos linfonodos $(\mathrm{pN})$ é determinante no uso de quimioterapia adjuvante $\mathrm{e}^{(3,4,9,23)}$. Vários estudos apontam um número mínimo de $\mathrm{LN}$ a analisar para que o estadiamento seja considerado adequado, principalmente na ausência de metástases $(2,3,7,11,13,15,18,32)$. Portanto, amostragem e dissecção adequadas de LN são cruciais na análise anatomopatológica e no estadiamento desses tumores, mas freqüentemente ficam aquém do desejável. Isso se deve a questões inerentes ao paciente, a variações anatômicas, à técnica cirúrgica, à ocorrência de tratamento neoadjuvante e à qualidade do exame anatomopatológico $(4,7,10,14,18,29,30)$. $\mathrm{O}$ número de $\mathrm{LN}$ pode ser menor em pacientes idosos ${ }^{(30)} \mathrm{e}$ depender do grau de resposta imunológica ${ }^{(10)}$. Características do tumor, como topografia e grau histológico, relacionam-se com o número de linfonodos comprometidos ${ }^{(30)}$. Em estudo anterior observamos variação de até $89 \%$ nas médias de $L N$ dissecados segundo a região, com a maior nos tumores do cólon ascendente (28,8 LN/caso) e a menor naqueles da flexura esplênica $\left(14,7 \mathrm{LN} /\right.$ caso) ${ }^{(29)}$. Essa variação pode depender não só de questões anatômicas, mas também da extensão do segmento ressecado. A técnica e a experiência do cirurgião são importantes e influenciam a qualidade do espécime ${ }^{(7,30)}$. Há registros de que cirurgiões gerais tendem a ressecar segmentos menores e abordar menos agressivamente as cadeias linfáticas que os especialistas ${ }^{(14)}$.

A qualidade da análise do patologista é fundamental, pois é necessário um exame minucioso e demorado na busca de $L N$, principalmente dos pequenos, que são a maioria. Há evidências que muitas das metástases linfonodais encontram-se em linfonodos com menos que $5 \mathrm{~mm}$ de diâmetro ${ }^{(7,10,16,31)}$ e que o estadiamento do CCR seria dependente não somente do número dos LN estudados, mas também de seu tamanho ${ }^{(10,31)}$ e sua localização ${ }^{(29,30)}$.
Ao que parece, o número de $\mathrm{LN}$ é tão ou mais importante do que o $\mathrm{pN}$. Estudos em casuísticas grandes demonstraram que a sobrevida piora com o aumento de LN positivos e que após controlar o número de $L N$ positivos a sobrevida melhora com o aumento do número de $\mathrm{LN}$ examinados $^{(22,25,27,30,35,36)}$. Discute-se se há um número mínimo de LN a examinar para que se possa ter segurança no estadiamento do paciente ${ }^{(11)}$. Diversos autores têm sugerido números que variam de seis a $18 \mathrm{LN}$ por espécime cirúrgico ${ }^{(2,3,7,15,33)}$ e o sistema TNM (T: tumor, $\mathrm{N}$ : linfonodo, M: metástase) recomenda a análise de 12 $\mathrm{LN}$, principalmente na ausência de metástases ${ }^{(34)}$. Todavia, Goldstein et al.(13) e Maurel et al. ${ }^{(26)}$ observaram que a probabilidade de estadiar um tumor como TNM estágio III tinha relação direta com o número de $L N$ examinados, atingindo um platô em 17 e $18 \mathrm{LN}$, respectivamente. Posteriormente, Goldstein ${ }^{(12)}$ observou que a probabilidade preditiva de se encontrar um LN positivo aumentava com o número de $L N$ examinados, sendo necessária a análise de $38 \mathrm{LN}$ para atingir $95 \%$ de probabilidade de obter um dado correto. A análise de 12 LN permitia apenas $25 \%$ de probabilidade preditiva de se encontrar um único LN comprometido, caso esse existisse. Joseph et al.(23) observaram, através de um modelo matemático, que seria necessária a análise de 29 a 42 LN para uma probabilidade de $85 \%$ de acerto no estadiamento de tumores pT4 e pT1, respectivamente.

A dissecção de $L N$ no cólon/reto é realizada tradicionalmente recortando-se o tecido adiposo pericolônico/perirretal em fatias finas, procurando-se os $\mathrm{LN}$ inicialmente por visão direta (LN grandes) e por percepção táctil palpando-se o tecido adiposo (para os $\mathrm{LN}$ menores). A percepção de $\mathrm{LN}$ menores de $4 \mathrm{~mm}$ é muito difícil e apesar de ser possível encontrar linfonodos bem pequenos (1 a $2 \mathrm{~mm}$ ), isso ocorre provavelmente por acaso. Métodos químicos de clareamento de gorduras têm sido propostos para melhorar a dissecção de LN. A maioria concorda que existe um ganho no número de $\mathrm{LN}$ dissecados, porém há controvérsias se o tempo e os recursos extras consumidos por esses processos justificam o seu emprego na rotina e mesmo se esse ganho numérico significa algum benefício para os pacientes $^{(5,16,17,21,24,32,33)}$. Entretanto, os poucos estudos que analisaram comparativamente o número de $\mathrm{LN}$ dissecados convencionalmente e com o uso de soluções clareadoras de gorduras (SC) evidenciaram mudanças de estadiamento dos pacientes ${ }^{(16,17,24,33)}$. O objetivo do presente estudo foi avaliar o impacto do uso de uma SC na detecção de LN e no estadiamento de pacientes com CCR. 


\section{Material e método}

\section{Material}

O estudo foi aprovado pelo Comitê de Ética em Pesquisa da Universidade Federal de Minas Gerais (UFMG) (protocolo $\left.n^{\circ} 108 / 05\right)$. Estudaram-se prospectivamente 74 peças cirúrgicas de pacientes operados por CCR no Instituto Alfa de Gastroenterologia do Hospital das Clínicas da UFMG, Belo Horizonte, Minas Gerais, no período de novembro de 2003 a setembro de 2005, não submetidos a tratamento neoadjuvante (rádio ou quimioterapia). Todos os pacientes foram operados pela mesma equipe cirúrgica de acordo com a rotina do serviço.

Os dados como gênero e idade dos pacientes, número de registro, sede do tumor e ocorrência de tratamento neoadjuvante (rádio ou quimioterapia) foram obtidos a partir das requisições de exame. Os dados relativos ao tipo histológico do tumor e o estadiamento patológico foram obtidos do laudo anatomopatológico. Todas as lâminas referentes aos $\mathrm{LN}$ dissecados pelo $\mathrm{MC}$ foram revistas, e os LN, medidos.

\section{Processamento dos espécimes}

Todos os espécimes cirúrgicos seguiram os procedimentos rotineiros de exame no serviço, de acordo com protocolo padronizado ${ }^{(28)}$. Os restos do tecido adiposo do mesocolo obtidos após a dissecção dos $\mathrm{LN}$ pelo MC, que seriam descartados junto com o espécime cirúrgico, foram submetidos a tratamento com SC, seguindo-se nova dissecção dos LN. A solução era composta por $65 \%$ de álcool etílico comercial a $95 \%, 20 \%$ de éter dietílico, $5 \%$ de ácido acético glacial e $10 \%$ de formol a $10 \%{ }^{(24)}$. O material foi mergulhado na solução em três banhos de 12 horas cada, usando-se volume suficiente para cobri-lo. Seguiram-se nova dissecção e processamento histopatológico rotineiro dos LN dissecados.

\section{Análise histológica e imuno-histoquímica, estadiamento e morfometria}

Após o exame histológico dos novos $\mathrm{LN}$ dissecados, os casos foram reestadiados quanto ao $\mathrm{pN}$, de acordo com o número total de $\mathrm{LN}$ dissecados (número de $\mathrm{LN}$ dissecados pelo $\mathrm{MC}+$ número de $\mathrm{LN}$ dissecados após o uso da SC). Realizou-se imuno-histoquímica pelo método da estreptavidina-biotina peroxidase ${ }^{(19)}$ em uma amostra composta de sete $L N$ escolhidos aleatoriamente entre os casos que apresentavam metástases após o uso da SC, a fim de veri- ficar se o uso da SC poderia interferir na imunomarcação. Testou-se o anti-soro mais comumente usado nas pesquisas de micrometástases, anticitoqueratina monoclonal AE1/AE3 (DAKO Corporation, Carpinteria, CA, USA) diluído a 1:30 e utilizou-se kit comercial Ultravision Large Volume Detection System (Labvision Corporation, Fremont, CA, USA).

Mediu-se o maior eixo dos LN usando-se uma lupa acoplada a régua de precisão $(0,2 \mathrm{~mm})$ para os $\mathrm{LN}$ maiores que $2 \mathrm{~mm}$ e através de morfometria computadorizada (Carl Zeiss - KS400, Alemanha) para LN menores. Determinou-se o número de $\mathrm{LN}$ maiores e menores que $4 \mathrm{~mm}$ dissecados pelos dois métodos e a medida em milímetros do menor e do maior $L N$ dissecado, nos grupos com e sem metástases.

\section{Análise estatística}

Os dados foram compilados em protocolo individual desenvolvido especificamente para o estudo, posteriormente informatizados e analisados em pacote estatístico SPSS, utilizando-se os testes de Wilcoxon, teste do $\chi^{2}$, teste-T pareado e teste- $T$ independente para comparar os resultados pré e pós-clareamento. O nível de significância foi estabelecido para $p<0,05$.

\section{Resultados}

\section{Casuística}

A casuística foi equilibrada em relação ao gênero, constando de 35 homens (47,3\%) e 39 mulheres $(52,7 \%)$ e não houve diferenças significativas quanto à média de idade (homens $64 \pm 12,7$ anos, mulheres $61 \pm 15,9$ anos; $p=0,5)$. Todos os CCRs eram adenocarcinomas. Predominaram os tumores moderadamente diferenciados $(n=60$; $81,1 \%)$; sete casos $(9,5 \%)$ eram adenocarcinomas mucinosos. Na maioria dos casos ( $n=51 ; 68,9 \%)$ os tumores eram $\mathrm{pT} 3$, sem diferenças significativas entre os gêneros $(p=0,1)$ (Figura 1). Em média os tumores tinham $76,5 \mathrm{~cm}^{3}$ $\left( \pm 100,7 \mathrm{~cm}^{3}\right)$ de volume; $5,1 \mathrm{~cm}( \pm 2,4 \mathrm{~cm})$ de dimensão longitudinal; $4,6 \mathrm{~cm}( \pm 2,1 \mathrm{~cm})$ de dimensão transversal e $2,4 \mathrm{~cm}( \pm 2,3 \mathrm{~cm})$ de profundidade, medidas estas sem variações expressivas entre os gêneros $(p \geq 0,2)$.

\section{Análise dos linfonodos}

Após os banhos na SC os LN eram facilmente visualizados, mesmo os muito pequenos (até $1 \mathrm{~mm}$ ), destacando-se 


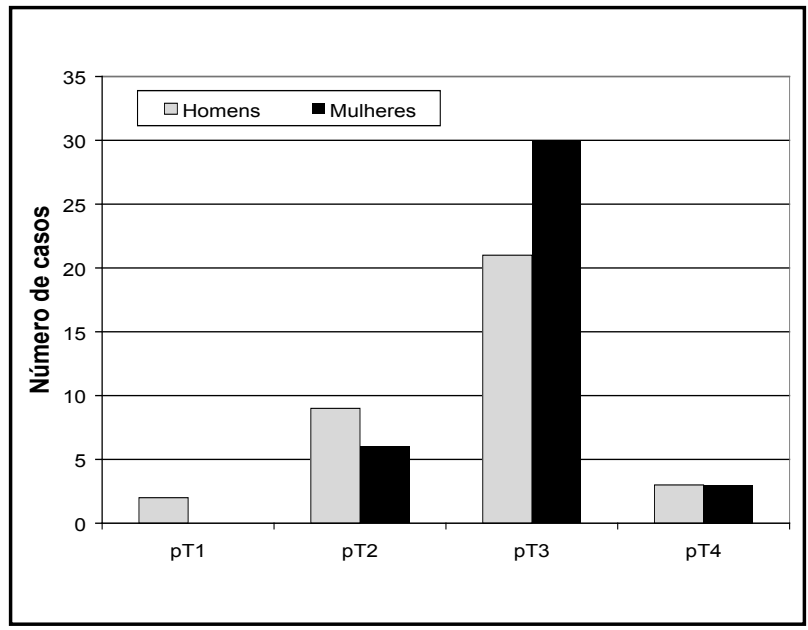

Figura 1 - Distribuição dos casos de carcinoma colorretal quanto ao pT

como pontos ou pequenos nódulos brancos sob o tecido adiposo amarelo-transparente (Figura 2). Não observamos alterações cromáticas nas colorações de rotina (HE) ou na imunomarcação no material submetido ao processo de clareamento. A imunomarcação pelo anti-soro anticitoqueratina AE1/AE3 foi fortemente positiva em toda a amostra testada de LN com metástases dissecados após o uso da SC (Figura 3).

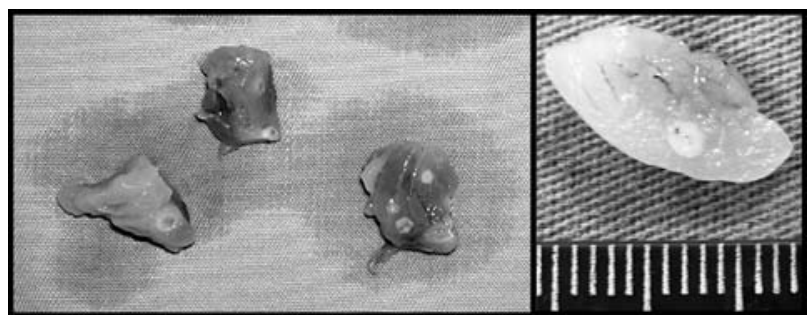

Figura 2 - Linfonodos destacados como pequenos nódulos brancos na gordura pericólica após processo de clareamento

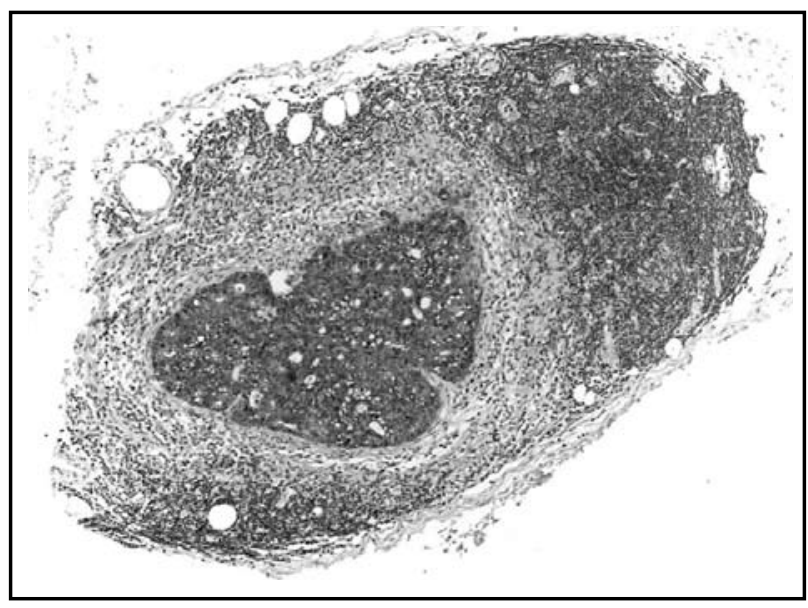

Figura 3 - Imunomarcação de metástases de adenocarcinoma colônico por pancitoqueratina (Anti-soro AE1/AE3; aumento original 100x)
Dissecaram-se $1.814 \mathrm{LN}$ pelo MC (24,5 LN/caso) e $1.355 \mathrm{LN}$ com o uso da SC (18,3 LN/caso), perfazendo um total de $3.167 \mathrm{LN}(42,8 \mathrm{LN} /$ caso). Cento e noventa e seis $\mathrm{LN}$ tinham metástases, dos quais $170(86,7 \%)$ foram obtidos pelo MC e $26(13,2 \%)$ após o tratamento com a SC.

Considerando os LN obtidos pelo MC, 1.068 (58,8\% - 14,4 LN/caso) tinham até $4 \mathrm{~mm}$ de diâmetro. Em média, o maior LN dissecado pelo MC tinha $8,87 \mathrm{~mm}( \pm 4,07 \mathrm{~mm}$; 3 a $23 \mathrm{~mm})$, e o menor, $1,41 \mathrm{~mm}( \pm 0,76 \mathrm{~mm} ; 0,4$ a 5 $\mathrm{mm}$ ). Considerando os $\mathrm{LN}$ dissecados após o tratamento com a SC, 1.261 (93\% - $17 \mathrm{LN} /$ caso) mediam até $4 \mathrm{~mm}$ de diâmetro. Em média, o maior $\mathrm{LN}$ dissecado após o uso da SC tinha $4,55 \mathrm{~mm}( \pm 1,67 \mathrm{~mm} ; 2,2$ a $11 \mathrm{~mm})$, e o menor, $1,05 \mathrm{~mm}( \pm 0,57 \mathrm{~mm} ; 0,2$ a $3,2 \mathrm{~mm})$. Entre os $170 \mathrm{LN}$ comprometidos obtidos pelo MC, 39 (22,9\%) mediam até $4 \mathrm{~mm}$ e 19 dos $26 \mathrm{LN}$ comprometidos (73\%) obtidos após uso da SC mediam até $4 \mathrm{~mm}(p=0)$. A média de diâmetro do menor LN com metástases encontrado após o uso da $\mathrm{SC}$ foi $3,16 \mathrm{~mm}( \pm 1,75 \mathrm{~mm} ; 1,4$ a $7 \mathrm{~mm})$. Estes resultados estão demonstrados na Tabela 1.

\section{Impacto do uso da solução clareadora}

\section{Número de linfonodos}

Após o uso da SC houve um acréscimo de $74,7 \%$ no número de $\mathrm{LN}$ dissecados $(p=0)$, um aumento de $118,1 \%$ na capacidade de dissecar $\mathrm{LN}$ pequenos $(p=0)$ e de $15,3 \%$ na capacidade de se encontrar metástases $(p=0,003)$ (Tabela 1$)$. Os menores $\mathrm{LN}$ foram aqueles dissecados após o clareamento. A média dos diâmetros dos menores $\mathrm{LN}$ dissecados após o uso da SC foi significativamente menor $(1,04 \mathrm{~mm})$ que daqueles dissecados pelo $\mathrm{MC}(1,4 \mathrm{~mm})(p=0)$.

O número de metástases linfonodais encontradas usando-se a SC foi maior quando havia metástases no MC (pelo menos um LN positivo identificado pelo $\mathrm{MC}$ ) $(p=0,007)$ (Tabela 2). Considerando-se o número total de $L N$ dissecados pelo $M C$, somente após a dissecção de 40 LN foram identificados $90 \%$ dos casos em que havia metástases linfonodais (Figura 4).

\section{Estadiamento}

Usando-se apenas o MC, seis (14\%) dos 43 casos estadiados como pN0 não haviam atingido o mínimo de $12 \mathrm{LN}$ dissecados, como recomendado pelo sistema TNM. Após o uso da SC apenas um caso (2,3\%) permaneceu nessa situação $(p=0,008)$ (Tabela 3). 


\section{Tabela 1 Número de linfonodos obtidos pelo método convencional e após o clareamento}

Número total de linfonodos dissecados

\begin{tabular}{|c|c|c|c|c|c|c|}
\hline & & Total & Metástases & Livres & $<4 \mathrm{~mm}$ & $\begin{array}{c}<4 \text { mm com } \\
\text { metástases }\end{array}$ \\
\hline \multirow[t]{2}{*}{ MC } & & 1.814 & 170 & 1.644 & 1.068 & 39 \\
\hline & Média & 24,51 & 2,3 & 22,21 & 14,43 & 1,34 \\
\hline \multirow[t]{2}{*}{ SC } & & 1.355 & 26 & 1.329 & 1.261 & 19 \\
\hline & Média & 18,31 & 0,35 & 17,96 & 17,04 & 1,26 \\
\hline \multirow[t]{2}{*}{$M C+S C$} & & 3.167 & 196 & 2.973 & 2.329 & 58 \\
\hline & Média & 42,8 & 2,65 & 40,17 & 31,47 & 1,75 \\
\hline Aumento (\%) & & 74,7 & 15,3 & 80,86 & 118 & 48,71 \\
\hline
\end{tabular}

MC: método convencional; SC: solução clareadora de gorduras.

Correlação entre a detecção de metástases linfonodais pelo método convencional e o número de $L N$ Tabela 2 positivos após 0 uso da SC

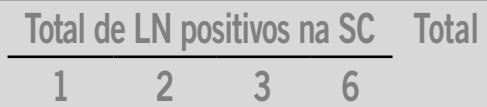

Metástases

pelo método

convencional

Não 22

4

( $n^{0}$ de casos)

$\begin{array}{llllll} & \operatorname{Sim} \quad 7 & 2 & 1 & 1 & 11 \\ \text { Total } & 9 & 4 & 1 & 1 & 15\end{array}$

LN: linfonodos; SC: solução clareadora.

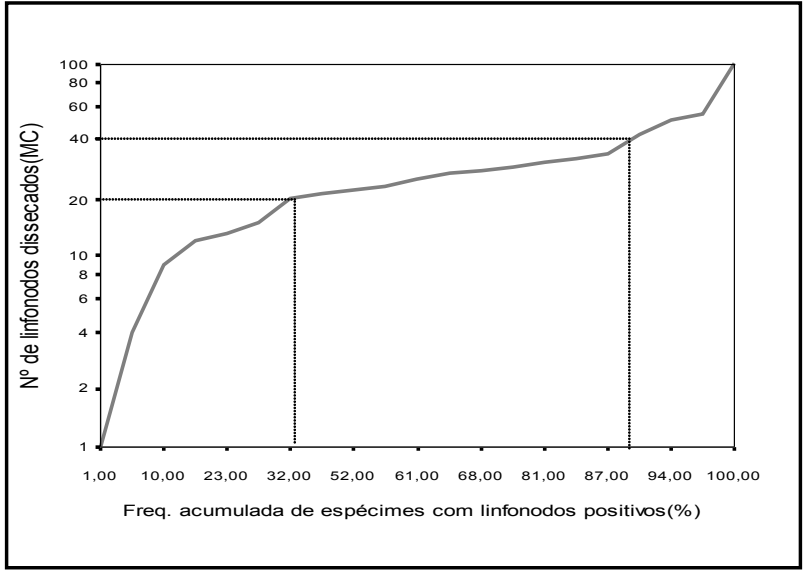

Figura 4 - Relação entre o número de linfonodos dissecados pelo método convencional (MC) e a freqüência acumulada de casos com metástases

Mudança de grupos de estadiamento do pN em relação ao ponto de corte (12 linfonodos), após Tabela 3 o uso da solução clareadora nos 43 carcinomas colorretais inicialmente estadiados como pNO

\begin{tabular}{|c|c|c|c|c|}
\hline & \multicolumn{2}{|c|}{ Após redissecção (solução clareadora) } & \multirow[t]{2}{*}{ Total } \\
\hline & & $<12$ linfonodos & $>12$ linfonodos & \\
\hline \multirow{2}{*}{$\begin{array}{l}\text { Dissecção } \\
\text { pelo método } \\
\text { convencional }\end{array}$} & $<12$ linfonodos & $1(16,7 \%)$ & $5(83,3 \%)$ & $6(14 \%)$ \\
\hline & $>12$ linfonodos & & 37 (100\%) & $37(86 \%)$ \\
\hline Total & & $1(2,3 \%)$ & $42(97,7 \%)$ & 43 (100\%) \\
\hline
\end{tabular}

Como podemos observar na Tabela 4, após a redissecção quatro casos (9,3\%) inicialmente pNO foram reestadiados como $\mathrm{pN} 1$ e um caso $(6,7 \%)$ inicialmente $\mathrm{pN} 1$ foi reestadiado como pN2. No total, o estadiamento foi alterado em cinco casos $(6,8 \%)(p=0,025)$ e quatro deles $(5,4 \%)$ mudaram de estágio II para estágio III. Analisando-se apenas os 43 casos inicialmente estadiados como $\mathrm{pNO}$, houve progressão significativa para pN1 $(p=0,046)$ após tratamento pela SC. As mudanças de estadiamento ocorreram em casos em que haviam sido dissecados de 12 a 20 LN (Tabela 5). 


\section{Mudanças de estadiamento detectadas após 0 uso da solução clareadora em 74 casos de câncer}

Tabela 4 colorretal

\begin{tabular}{ccc}
\multicolumn{2}{l}{ Estadiamento após redissecção (solução clareadora) } & Total \\
pNO & pN1
\end{tabular}

\begin{tabular}{|c|c|c|c|c|c|}
\hline \multirow{3}{*}{$\begin{array}{l}\text { Estadiamento } \\
\text { pelo método } \\
\text { convencional }\end{array}$} & pNO & $39 \operatorname{casos}(90,7 \%)$ & $4 \operatorname{casos}(9,3 \%)$ & & $43 \operatorname{cas} 0 \mathrm{~s}(100 \%)$ \\
\hline & $\mathrm{pN1}$ & & $14 \operatorname{casos}(93,3 \%)$ & 1 caso $(6,7 \%)$ & 15 casos $(100 \%)$ \\
\hline & pN2 & & & 16 casos $(100 \%)$ & 16 casos $(100 \%)$ \\
\hline Total & & $39 \operatorname{casos}(52,7 \%)$ & $18 \operatorname{casos}(24,3 \%)$ & $17 \operatorname{casos}(23 \%)$ & 74 casos $(100 \%)$ \\
\hline
\end{tabular}

Mudanças de estadiamento em relação ao número inicial de linfonodos dissecados

Tabela 5 convencionalmente e ao número final de linfonodos após 0 uso da solução clareadora

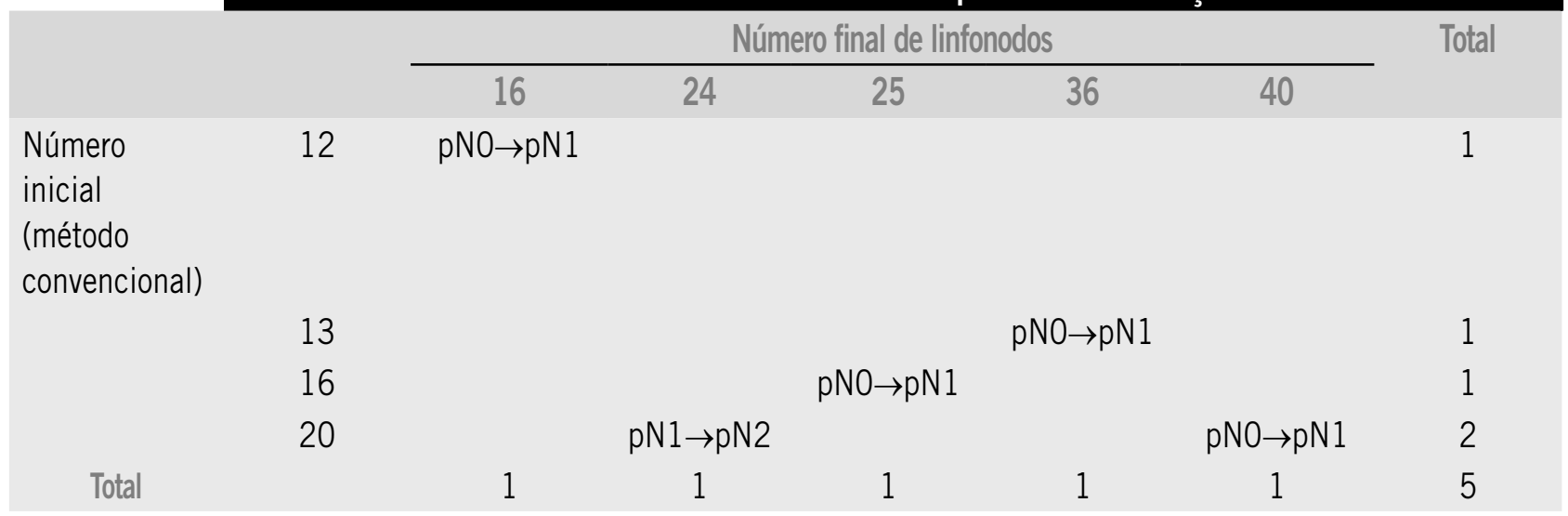

\section{Discussão}

É reconhecido que o $\mathrm{pT}$ e o $\mathrm{pN}$, juntamente com a angioinvasão e a presença de tumor residual, são fatores com comprovada importância prognóstica, geralmente usados no manejo dos pacientes portadores de $\mathrm{CCR}^{(9)}$. A presença de metástases em $L N$ regionais é, isoladamente, o fator prognóstico mais importante do $\mathrm{CCR}^{(23)}$ e o principal determinante no uso de quimioterapia ${ }^{(3,4,9,23)}$. Idealmente, todos os $\mathrm{LN}$ deveriam ser retirados pelo cirurgião e examinados pelo patologista, o que em termos práticos é impossível pelo método convencional baseado na palpação sistemática de cortes finos do tecido adiposo pericólico. Este método é demorado e ineficaz na dissecção de $L N$ pequenos, freqüentemente comprometidos ${ }^{(1,10,16)}$. A ampla variação no número de $L N$ dissecados registrada na literatura demonstra claramente que esse método é inadequado e se recomendam novos métodos de dissecção de $L N$, com destaque para os métodos químicos $(4,17,24,32,33)$. Segundo alguns autores, a avaliação quantitativa correta do envolvimento metastático de LN somente seria possível usando-se técnicas de clareamento de gorduras ${ }^{(32)}$.

Testamos a SC descrita por Koren et al. ${ }^{(24)}$ com pequenas modificações no tempo dos banhos e na quantidade de solução usada, visando adequação à rotina do nosso serviço. Eliminamos o viés da técnica cirúrgica, do cirurgião e do patologista, porque todos os casos foram operados pela mesma equipe de cirurgiões especialistas, a dissecção convencional foi feita pela mesma equipe de residentes de patologia sob supervisão da mesma especialista (AMN) e a redissecção foi feita pelo mesmo patologista (RX). A SC não contém produtos tóxicos como xilol, empregados nas SC usadas por outros au- 


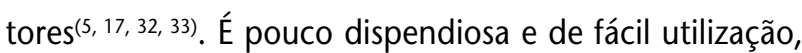
pois não necessita de nenhum dispositivo específico, sendo composta de insumos presentes ou facilmente adquiríveis em um laboratório de patologia. Apesar de representar um custo adicional de $\mathrm{R} \$ 1$ a $\mathrm{R} \$ 2$ por espécime, esse valor é compensado com lucro pelo aumento de eficiência e qualidade na dissecção e pela grande economia de tempo do examinador. Seu uso não interferiu na qualidade das colorações de rotina, confirmando o observado por Koren et al. ${ }^{(24)}$, nem no estudo imuno-histoquímico para citoqueratina.

No nosso serviço a média de $\mathrm{LN}$ dissecados pelo $\mathrm{MC}$ é alta (21 LN/caso) ${ }^{(29)}$ e neste estudo foi $24,5 \mathrm{LN} /$ caso, o que é o dobro do mínimo recomendado pelo sistema $\mathrm{TNM}^{(34)}$ e está acima do descrito na literatura, com variações de 3,1 a $21 \mathrm{LN} /$ caso (Tabela 6). Além disso, foi uma dissecção minuciosa com boa qualidade dentro das limitações do método, uma vez que 59\% dos LN tinham menos de $4 \mathrm{~mm}$, valor comparável aos obtidos por outros $(49 \%-70 \%)^{(10,17,32)}$. Os poucos $\mathrm{LN}$ maiores que 4 $\mathrm{mm}$ recuperados posteriormente com o uso da SC, eram afilados e estavam justapostos a vasos sanguíneos, o que provavelmente ocasionou sua perda durante a dissecção convencional. O uso da SC implicou num ganho de $74 \%$ na eficiência da dissecção com uma média final de 42,8 LN/caso. Em números absolutos esse ganho é alto, comparável aos valores mais altos obtidos por outros autores utilizando métodos auxiliares de dissecção ${ }^{(16,17,32)}$. Hida et al.(17) dissecaram 76 LN/caso, e Herreras-Ornelas et al.(16), $52 \mathrm{LN} /$ caso, porém esses autores usaram métodos muito complicados, dispendiosos e demorados (em torno de duas semanas), talvez apenas admissíveis em protocolos de pesquisa.

É notório que o ganho no número de LN promovido pelo uso da SC foi devido ao aumento na capacidade de se encontrarem LN pequenos, que eram a maioria. Como observado por outros autores, que empregaram variadas técnicas de clareamento, a dissecção facilitada pelo uso da visão permite dissecar $\mathrm{LN}$ tão pequenos como $1 \mathrm{~mm}$, eliminando a perda de $\mathrm{LN}$ pequenos ${ }^{(16,17,32,33)}$. Hida et al. ${ }^{(17)}$ e Schimitz-Moorman et al. ${ }^{(32)}$ relataram que $77 \%$ e $73,4 \%$ dos $\mathrm{LN}$ dissecados após o clareamento tinham até $4 \mathrm{~mm}$ de diâmetro contra $49 \%$ dos LN obtidos pelo MC. Nossos dados mostraram predominância de LN de até $4 \mathrm{~mm}$ de diâmetro, o que representou um ganho de $118 \%$ em relação ao MC. Observamos que em $20 \%$ dos casos com metástases o maior LN media até $4 \mathrm{~mm}$. Esse índice é ligeiramente inferior ao observado por Cserni (33\%), que contudo considerou como ponto de corte LN menores que $5 \mathrm{~mm}^{(10)}$. Dezenove dos $26 \mathrm{LN}$ comprometidos obtidos após o uso da SC e os $\mathrm{LN}$ comprometidos nos quatro casos que mudaram o estadiamento de pN0 para pN1 mediam até 4 $\mathrm{mm}$. Esses dados reforçam a importância da dissecção dos LN pequenos enfatizada em outros estudos $(10,16)$.

$\mathrm{O}$ aumento no número de $\mathrm{LN}$ dissecados refletiu-se no aumento de $15,3 \%$ de LN comprometidos. Esse percentual é significativo e semelhante aos $17 \%$ relatados por Cohen et al. ${ }^{(5)}$, porém muito menor do que o observado por Scott e $\mathrm{Grace}^{(33)}$, que encontraram metástases adicionais em 73,5\%, e Schimitz-Moorman et al. ${ }^{(32)}$, que relataram $89,3 \%$ de metástases nos $\mathrm{LN}$ dissecados por método auxiliar. A diferença se deve, no entanto, à baixa eficiência da

\section{Tabela 6}

\section{Séries}

Presente série

Pereira Jr. et al.(29)

Cohen et al. ${ }^{(5)}$

Joseph et al.(23)

Goldstein $^{(12)}$

Hida et al.(17)

Cserni(10)

Scott e Grace(33)

Schimitz-Moorman et al. ${ }^{(32)}$
Dissecção média de linfonodos (LN) pelo método convencional em casos de carcinoma colorretal, registrada na literatura e em nossa casuística
24,5

21

21

18,5

18,4

18,1

12,4

6,1

3,1 
dissecção convencional demonstrada pelos autores (seis e três $\mathrm{LN} /$ caso, respectivamente) (Tabela 6). Nossos dados corroboram os de outros estudos, que demonstraram que quanto mais LN eram examinados, mais metástases eram identificadas ${ }^{(13)}$. Le Voyer et al.(25) observaram que a análise de mais $L N$, comprometidos ou não, estava associada a maior sobrevida dos pacientes. Segundo alguns autores, a dissecção de muitos LN diminuiria a massa residual da neoplasia, podendo aumentar a eficácia de quimioterapia adjuvante, com conseqüente aumento da sobrevida desses pacientes ${ }^{(15,23)}$. A comprovação dos benefícios diretos da dissecção de $L N$ em nossos pacientes só poderá ser feita futuramente com análise de sobrevida.

O uso da SC permitiu a detecção de mais metástases em 15 casos, dos quais cinco mudaram o estadiamento: quatro de $\mathrm{pN} 0$ para pN1 e um de pN1 para pN2. Portanto, quatro dos 43 casos inicialmente estadiados como pNo $(9,3 \%)$ eram casos pN1 subestadiados. Além disso, foi possível estadiar cinco dos seis casos (83,3\%) que inicialmente não haviam atingido o mínimo de $12 \mathrm{LN}$ dissecados sugerido pelo sistema TNM. Não há consenso quanto ao número ideal de $\mathrm{LN}$ a dissecar, principalmente em casos pN0, para que se possa considerar o paciente com CCR adequadamente estadiado. Existem sugestões de valores entre sete e 18 linfonodos $^{(13,15,38)}$, e o sistema TNM ${ }^{(34)}$ sugere 12 LN. No entanto, em nosso estudo todos os casos que mudaram de estadiamento após o uso da SC tinham 12-20 LN dissecados pelo MC (Tabela 5). Além disso, apenas após a análise de 40 LN identificamos $90 \%$ dos casos com LN comprometidos (Figura 4). Esses resultados se correlacionam com os de Joseph et al. ${ }^{(23)}$, que com o uso de um modelo matemático sugeriram valores em torno de $40 \mathrm{LN}$ para CCR pT1-pT3 e em torno de 30 para pT4. Le Voyer et al. ${ }^{(25)}$ demonstraram que, mesmo para os grupos de pacientes pN1 e pN2 a sobrevida geral em cinco anos foi melhor (90\% e $71 \%$ ) nos pacientes em que haviam sido examinados mais de 40 $\mathrm{LN}$ e mais de $35 \mathrm{LN}$, respectivamente, havendo ainda uma melhora contínua com a evolução até 10 anos. Com base nesses resultados, concordamos com Goldstein ${ }^{(13)}$, quando este afirma que não existe um número mínimo a ser alcançado para se obter um estadiamento correto ou acurado de todos os pacientes, e com a equação de Bilchik ${ }^{(1)}$ : More (nodes) + more (analysis) $=$ less (mortality).

\section{Conclusão}

Nossos dados demonstram que o exame de muitos $L N$ e principalmente de LN pequenos aumenta a probabilidade de se encontrarem metástases e o grau de confiança do estadiamento dos pacientes com CCR. O mínimo de 12 LN dissecados sugerido pelo sistema TNM não garante o estadiamento adequado em casos de pNO. Uma solução de clareamento de gorduras simples de usar e pouco dispendiosa pode ser de grande ajuda nesse propósito.

\section{Agradecimentos}

Agradecemos ao Prof. Dr. Konradin Metze, coordenador do Laboratório de Anatomia Patológica Experimental no Núcleo de Medicina e Cirurgia Experimental da Universidade Estadual de Campinas (UNICAMP), pela contribuição nas análises estatísticas e na discussão dos resultados, e aos colegas patologistas que realizaram a análise anatomopatológica convencional: Cynthia Koeppel Berenstein, Daniel Ribeiro Moreira, Frederico Henrique Corrêa de Melo, Luciana de Paula Lima Gazzola, Marcelo Antônio Pascoal Xavier e Mário Félix Richard de Lima.

\section{Referências}

1. BILCHIK, A. More (nodes) + more (analysis) = less (mortality): challenging the therapeutic equation for early-stage colon cancer. Ann Surg Oncol, v. 10, n. 3, p. 203-5, 2003.

2. CAPLIN, S. et al. For patients with Duke's B (TNM stage II) colorectal carcinoma, examination of six or fewer lymph nodes is related to poor prognosis. Cancer, v. 83, p. 666-72, 1998.

3. CIANCHI, F. et al. Lymph node recovery from colorectal tumor specimens: recommendation for a minimum number of lymph nodes to be examined. World J Surg, v. 26, n. 3, p. 384-9, 2002.
4. COHEN, A. M. et al. Adjuvant therapy for colorectal cancer. Curr Probl Cancer, v. 22, n. 1, p. 11-77, 1998.

5. COHEN, S. M. et al. Effect of xylene clearance of mesenteric fat on harvest of lymph nodes after colonic resection. Eur J Surg, v. 160, n. 12, p. 693-7, 1994.

6. COMPTON, C. C. Colorectal carcinoma: diagnostic, prognostic, and molecular features. Mod Pathol, v. 16, n. 4, p. 376-88, 2003.

7. COMPTON, C. C. Pathologic staging of colorectal cancer: an advanced users' guide. Pathology Case Reviews, v. 9, p. 150-62, 2004. 
8. COMPTON, C. C. Updated protocol for the examination of specimens from patients with carcinomas of the colon and rectum, excluding carcinoid tumors, lymphomas, sarcomas, and tumors of the vermiform appendix: a basis for checklists. Cancer Committee. Arch Pathol Lab Med, v. 124, n. 7, p. 1016-25, 2000.

9. COMPTON, C. C. et al. Prognostic factors in colorectal cancer. College of American Pathologists Consensus Statement 1999. Arch Pathol Lab Med, v. 124, n. 7, p. 979-94, 2000.

10. CSERNI, G. The influence of nodal size on the staging of colorectal carcinomas. J Clin Pathol, v. 55, n. 5, p. 386-90, 2002.

11. CSERNI, G. et al. Is there a minimum number of lymph nodes that should be histologically assessed for a reliable nodal staging of T3NOMO colorectal carcinomas? J Surg Oncol, v. 81, n. 2, p. 63-9, 2002.

12. GOLDSTEIN, N. S. Lymph node recoveries from 2427 pT3 colorectal resection specimens spanning 45 years: recommendations for a minimum number of recovered lymph nodes based on predictive probabilities. Am J Surg Pathol, v. 26, n. 2, p. 179-89, 2002.

13. GOLDSTEIN, N. S. et al. Lymph node recovery from colorectal resection specimens removed for adenocarcinoma. Trends over time and a recommendation for a minimum number of lymph nodes to be recovered. Am J Clin Pathol, v. 106, n. 2, p. 209-16, 1996.

14. HERMANEK, P. Impact of surgeon's technique on outcome after treatment of rectal carcinoma. Dis Colon Rectum, v. 42, n. 5, p. 559-62, 1999.

15. HERNANZ, F. et al. Colorectal adenocarcinoma: quality of the assessment of lymph node metastases. Dis Colon Rectum, v. 37, n. 4, p. 373-7, 1994.

16. HERRERA-ORNELAS, L. et al. Metastases in small lymph nodes from colon cancer. Arch Surg, v. 122, n. 11, p. 1253-6, 1987.

17. HIDA, J. et al. Metastases from carcinoma of the colon and rectum detected in small lymph nodes by the clearing method. J Am Coll Surg, v. 178, n. 3, p. 223-8, 1994.

18. HORZIC, M.; KOPLJAR, M. Minimal number of lymph nodes that need to be examined for adequate staging of colorectal cancer: factors influencing lymph node harvest. Hepatogastroenterology, v. 52, n. 61, p. 86-9, 2005.

19. HSU, S. M. et al. Use of avidin-biotin-peroxidase complex $(\mathrm{ABC})$ in immunoperoxidase techniques: a comparison between $A B C$ and unlabeled antibody (PAP) procedures. J Histochem Cytochem, v. 29, n. 4, p. 577-80, 1981.

20. INSTITUTO NACIONAL DO CÂNCER. São Paulo: Estimativas da incidência e mortalidade por câncer no Brasil 2008. Disponível em: http://www.inca.gov.br/.

21. JASS, J. R. et al. Fat clearance method versus manual dissection of lymph nodes in specimens of rectal cancer. Int J Colorectal Dis, v. 1, n. 3, p. 155-6, 1986.

22. JOHNSON, P. M. et al. Increasing negative lymph node count is independently associated with improved long- term survival in stage IIIB and IIIC colon cancer. J Clin Oncol, v. 24, n. 22, p. 3570-5, 2006.

23. JOSEPH, N. E. et al. Accuracy of determining nodal negativity in colorectal cancer on the basis of the number of nodes retrieved on resection. Ann Surg Oncol, v. 10, n. 3, p. 213-8, 2003.

24. KOREN, R. et al. Lymph node-revealing solution: simple new method for detecting minute lymph nodes in colon carcinoma. Dis Colon Rectum, v. 40, n. 4, p. 407-10, 1997.

25. Le VOYER, T. E. et al. Colon cancer survival is associated with increasing number of lymph nodes analyzed: a secondary survey of intergroup trial INT-0089. J Clin Oncol, v. 21, n. 15, p. 2912-9, 2003.

26. MAUREL, J. et al. Lymph node harvest reporting in patients with carcinoma of the large bowel: a French populationbased study. Cancer, v. 82, p. 1482-6, 1998.

27. MERRIE, A. E. et al. Prognostic significance of occult metastases in colon cancer. Dis Colon Rectum, v. 46, n. 2, p. 221-31, 2003.

28. PEREIRA Jr., T. et al. Câncer colorretal: análise anatomopatológica de 476 colectomias consecutivas em Belo Horizonte (MG). J Bras Patol Med Lab, v. 41, n. 3, p. 175-84, 2005.

29. PEREIRA Jr, T. et al. Lymph node evaluation in colorectal cancer. Arq Gastroenterol, v. 43, n. 2, p. 89-93, 2006.

30. PRANDI, M. et al. Prognostic evaluation of stage $B$ colon cancer patients is improved by an adequate lymphadenectomy: results of a secondary analysis of a large scale adjuvant trial. Ann Surg, v. 235, n. 4, p. 458-63, 2002.

31. RODRIGUEZ-BIGAS, M. A. et al. Clinical significance of colorectal cancer: metastases in lymph nodes $<5 \mathrm{~mm}$ in size. Ann Surg Oncol, v. 3, n. 2, p. 124-30, 1996.

32. SCHMITZ-MOORMANN, P. et al. Patho-anatomical demonstration of lymph node metastases in a surgical specimen. Pathol Res Pract, v. 174, n. 4, p. 403-11, 1982.

33. SCOTT, K. W.; GRACE, R. H. Detection of lymph node metastases in colorectal carcinoma before and after fat clearance. Br J Surg, v. 76, n. 11, p. 1165-7, 1989.

34. SOBIN, L. H., WITTEKIND, C.H. Colon and rectum. In: UICC (Ed.). TNM classification of malignant tumors. $6^{\text {th }}$ ed. New York: Willy-Liss, 2002. p. 72-6.

35. SWANSON, R. S. etal. The prognosis of T3NO colon cancer is dependent on the number of lymph nodes examined. Ann Surg Oncol, v. 10, n. 1, p. 65-71, 2003.

36. TEPPER, J. E. et al. Impact of number of nodes retrieved on outcome in patients with rectal cancer. J Clin Oncol, v. 19 , n. 1, p. 157-63, 2001.

37. WHO. International Agency for Research on Cancer. Globocan 2002. Disponível em: http://www-dep.iarc.fr.

38. WONG, J. H. et al. Number of nodes examined and staging accuracy in colorectal carcinoma. J Clin Oncol, v. 17, n. 9, p. 2896-900, 1999. 